\title{
Calculation of Ship Collision Risk Index Based on Adaptive Fuzzy Neural Network
}

\author{
Chunze Li, Wei Li and Jun Ning* \\ Navigation College, Dalian Maritime University, Dalian 116026, China \\ ${ }^{*}$ Corresponding author
}

\begin{abstract}
This paper combines adaptive fuzzy system and neural network to construct an adaptive fuzzy neural network. Using DCPA, TCPA raw data, speed (V), heading (C), angle (Q), distance (D) as the training input of the network, and comparing the learning results of BP neural network and adaptive fuzzy neural network to predict Ship collision risk index. The results show that the adaptive fuzzy neural network has good prediction results.
\end{abstract}

Keywords—adaptive fuzzy neural network; BP neural network; ship collision risk Index

\section{INTRODUCTION}

Ship collision accidents are the focus of attention due to the harm they bring. How to avoid the collision of ships has always been the focus of researchers. In the process of researching ship automation, how to determine whether a ship has a collision risk and the size of a collision risk is a prerequisite for automated research. As to how to determine the collision risk of a ship, the Japanese scholar Imazu Kamima proposed the concept of ship collision risk index in 1983 and conducted detailed analysis [1].On this basis, scholars from all over the world have also begun to conduct in-depth studies on the risk of collision of ships. For the study of ship collision risk index, DCPA, TCPA weighted method, fuzzy reasoning, genetic algorithm, artificial neural network method and other methods are mainly used in recent years[2].

In the method of calculating the Collision Risk Index of a ship, the neural network method is highly favored by scholars due to its high nonlinear mapping ability, self-organization and self-adaptability, etc. It can perform complex logic operations and nonlinear mapping. There are more scholars using artificial neural network methods to calculate the Collision Risk Index of ships [3, 4].

Due to the ambiguity of collision risk, many scholars use fuzzy theory to calculate the collision risk of the ship [5, 6].Because in fuzzy systems, the design of fuzzy sets, membership functions, and fuzzy rules is based on empirical knowledge. Therefore, there is a great degree of subjectivity in algorithm design.Therefore, scholars began to combine the learning ability of neural networks with fuzzy systems to construct fuzzy neural network algorithms [7, 8]. Fuzzification, fuzzy reasoning, and precise calculation of fuzzy systems are expressed through distributed neural networks to realize the ability of self-organizing and self-learning of fuzzy systems.
The conventional neural network is given fuzzy inputs and fuzzy weights to further promote the neural network.

Although the use of an adaptive fuzzy neural network to calculate the CRI of ships is not the first proposed in this paper, the reference $[7,8]$ uses the fuzzy neural network and the reference [9] uses the BP neural network to calculate the CRI, using DCPA and TCPA as neural network's two input values to calculate the CRI.DCPA and TCPA are the most direct and main judging criteria for the CRI of ships. However, if fuzzy neural network is only based on DCPA and TCPA, due to fewer input factors, neural network can not cover the, various knowledge problems.

\section{A. BP Neural Network}

In this paper, constructed the BP neural network structure to determine the collision risk of the ship. The ship collision risk index(CRI) is determined by the speed (V), heading (C), angle (Q), and distance (D) as input to the network. Select the 4-9-1 network structure as in reference [3]. The network structure is as shown in Fig. 1.

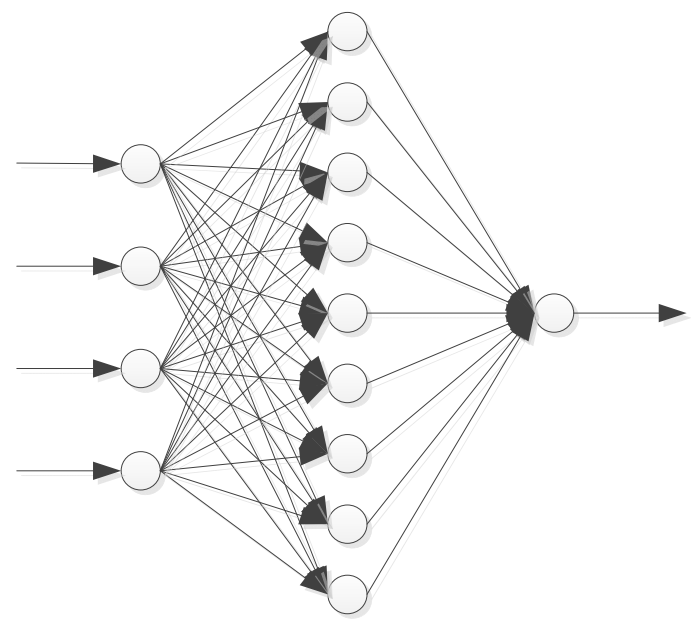

FIGURE I. BP NEURAL NETWORK STRUCTURE

The BP neural network calculates the hidden layer output and the output of the neural network of the neural network through forward propagation. In the calculation process, the output of the error is used to control the progress of the network. When the error meets the desired requirement, the algorithm 
ends. Otherwise, the connection weight of the network is readjusted by back propagation until the error is minimized[10].

Using the error back-propagation to train the BP learning algorithm, the neural network is like a "black box", which stores experts' experiences and reasoning mechanisms for multi-attribute decision making. When making a decision on a new target, new attribute features are input into the neural network, and the neural network will give an output evaluation value.

\section{ADAPTIVE FUZZY NEURAL NETWORK}

\section{A. Adaptive Fuzzy System}

The neural network algorithm establishes a T-S type FIS by learning, associating, and inferring a large amount of known data. The fuzzy rules and membership function parameters are determined by neural networks using "Back-Propagation” [12]. The network structure of adaptive fuzzy neural network is shown in Fig. 2.

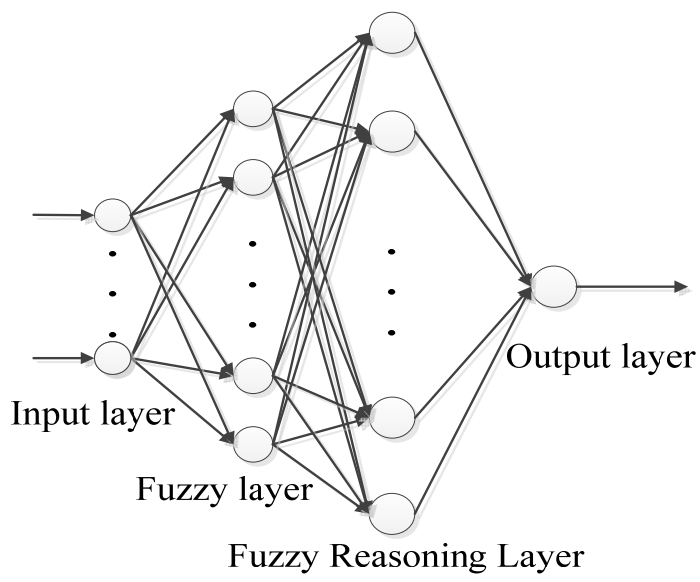

FIGURE II. ADAPTIVE FUZZY NEURAL NETWORK STRUCTURE

The T-S fuzzy system has strong self-adaptive ability, can continuously self-renew during the process of reasoning, and continuously repair the membership function. When defining it, use the "if-then" rule formal definition, set the rule is $R^{i}$, then the reasoning process is as follows:

$$
R^{i} \text { : if } x_{i} \text { is } A_{1}^{i}, \cdots, x_{k} \text { is } A_{k}^{i} \text { then } y_{i}=p_{0}^{i}+p_{1}^{i} x_{1}+\ldots+P_{k}^{i} x_{k}
$$

Where, $A_{j}^{i}$ is the fuzzy set of the fuzzy system; $P_{j}^{i}(j=1,2, \cdots, n)$ is the fuzzy system parameter; $y_{j}$ is the output according to the fuzzy rule; when the adaptive fuzzy is inferred, because the input part is fuzzy, and the output part is determined, the fuzzy inference process represents a linear relationship.

Assuming that the input value is $x=\left[x_{1}, x_{2}, \cdots, x_{k}\right]$, the membership of the input variable $x_{j}$ is calculated according to the fuzzy rules:

$$
\mu A_{j}^{i}=\exp \left(-\left(x_{j}-c_{j}^{i}\right)^{2} / b_{j}^{i}\right) \quad k=1,2, \cdots, k \quad i=1,2, \cdots, n
$$

In the formula, $c_{j}^{i}, b_{j}^{i}$ are the center and width of the membership function; $k$ is the input parameter; $n$ is the number of fuzzy subsets.

The membership degree is calculated in a fuzzy manner and the fuzzy operator is used as a concatenation operator:

$$
\omega^{i}=u A_{j}^{1}\left(x_{1}\right) * u A_{j}^{2}\left(x_{2}\right) * \ldots * u A_{j}^{k}\left(x_{k}\right) \quad i=1,2, \cdots, n
$$

Calculate the output value of the fuzzy model based on the fuzzy calculation result:

$$
y_{i}=\sum_{i=1}^{n} \omega^{i}\left(p_{0}^{i}+p_{1}^{i} x_{1}+\ldots+P_{k}^{i} x_{k}\right) / \sum_{i=1}^{n} \omega^{i}
$$

\section{B. Adaptive Fuzzy Neural Network}

The neural network and adaptive fuzzy system are combined to form an adaptive fuzzy neural network. The T-S fuzzy neural network is divided into an input layer, a fuzzification layer, a fuzzy rule calculation layer, and an output layer. The input layer is connected to the input vector $x_{i}$, and the number of nodes is the same as the dimension of the input vector. The fuzzy layer uses a membership function (refer with: Eq. 11) to fuzzily the input value to obtain a fuzzy membership value $\mu$. The fuzzy rule calculation layer uses the fuzzy continuous(refer with: Eq. 12)to calculate $\omega$. The output layer uses Eq. 13 to calculate the output of the fuzzy neural network. The network flow is shown in Fig. 3. 


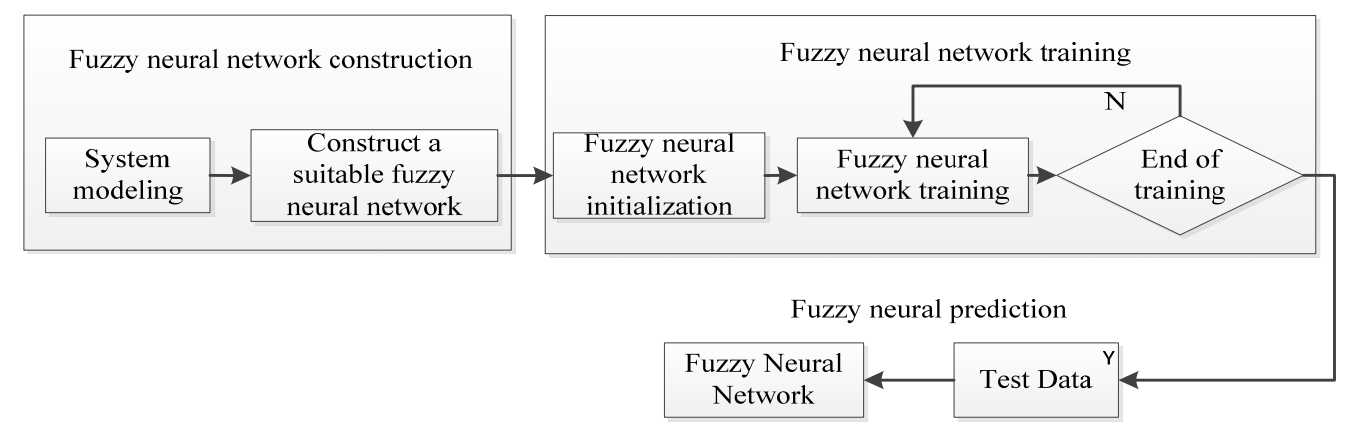

FIGURE III. FUZZY NEURAL NETWORK PROCESS

The algorithm of the fuzzy neural network algorithm is as follows:

(1) Error calculation

$$
e=\frac{1}{2}\left(y_{d}-y_{c}\right)^{2}
$$

Where: $y_{d}$ is the expected output of the network; $y_{e}$ is the actual output of the network; $e$ is the expected output and the actual output error.

(2) Coefficient correction

$$
\begin{gathered}
c_{j}^{i}(k)=c_{j}^{i}(k-1)-\beta \frac{\partial e}{\partial p_{j}^{i}} \\
b_{j}^{i}(k)=b_{j}^{i}(k-1)-\beta \frac{\partial e}{\partial b_{j}^{i}}
\end{gathered}
$$

Where $c_{j}^{i}$ and $b_{j}^{i}$ are the center and width of the membership function, respectively.

\section{CASE STUDY}

This paper uses the matlab to realize the above-mentioned adaptive fuzzy neural network. In this paper, we set the ship's speed to be $14 \mathrm{kn}$ and the heading to $60^{\circ}$. Then we train the speed(V), heading(C), angle(Q) and distance(D) of 80 randomly selected ships.Use BP network and adaptive neural network to forecast the collision risk of ships and compare them.

\section{A. BP Neural Network Training Results}

In the prediction of ship collision risk index (CRI) through network training, due to the different dimension levels of the four inputs, a normalization process is needed, Normalized reference reference [4], due to the headings in this article are true headings and angles' range is $360^{\circ}$. Therefore, the following normalization process is performed:

$$
\left(x_{1}, x_{2}, x_{3}, x_{4}\right)=\left(V / 50, C / 360^{\circ}, Q / 360^{\circ}, D / 12\right)
$$

After a lot of training, the neural network training has a good fitting effect, the $\mathrm{R}$ value is 0.96926 , and the neural network fitting effect figure is shown in Fig. 4.

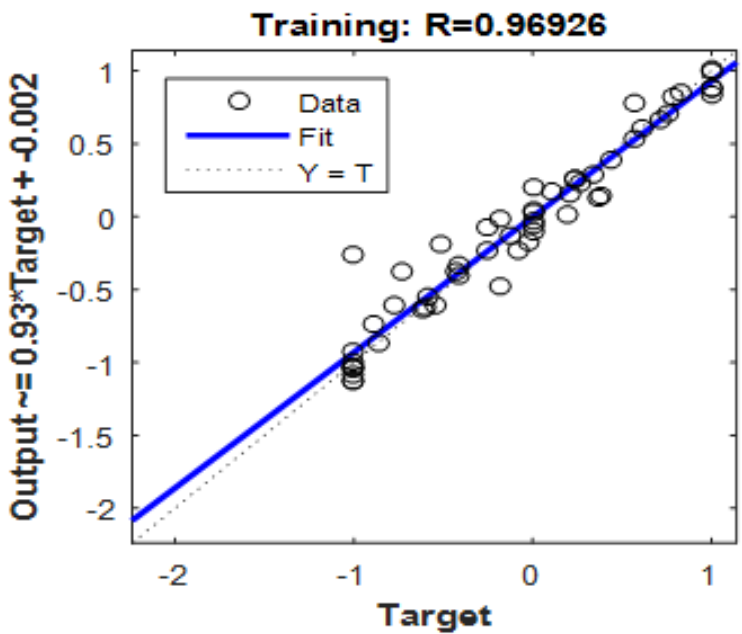

FIGURE IV. NEURAL NETWORK TRAINING FITTING RESULTS

Using the trained BP neural network to confirm the collision risk of the ship, the prediction error of the BP neural network is 0.07 , and the prediction result of the BP neural network is good. The forecast effect chart is shown in Fig. 5 and Fig. 6 


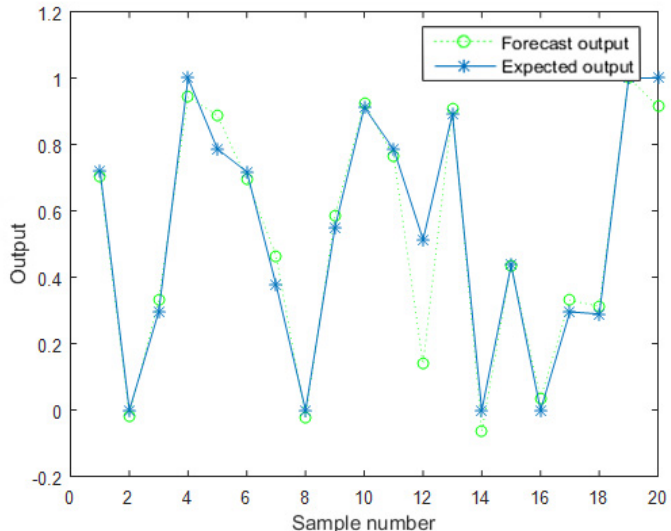

FIGURE V. BP NEURAL NETWORK FORECAST RESULT

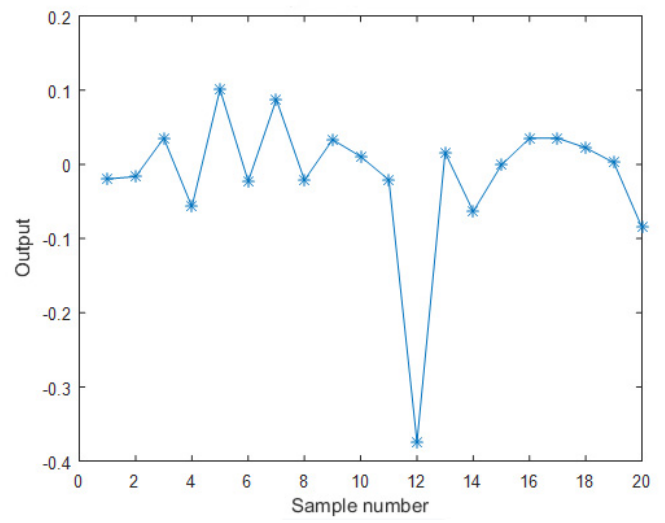

FIGURE VI. ERROR CURVE

\section{B. Adaptive Fuzzy Neural Network Training Results}

During the training of the adaptive fuzzy neural network, 80 sets of data were trained for 300 times, and the adaptive neural network established a 4-54-1 neural network structure, making the network error is 0.003 . The network training effect is shown in Fig. 7. And using the trained adaptive fuzzy neural network to predict the other 20 groups of data, the prediction results are shown in Fig. 8.

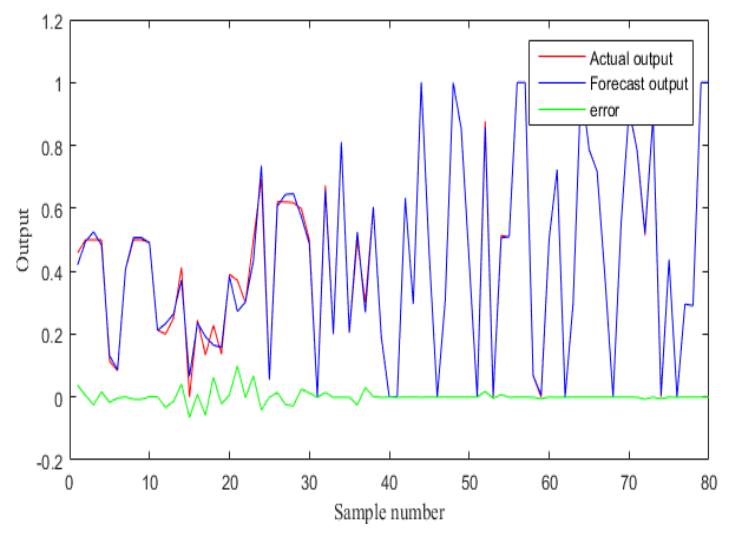

FIGURE VII. TRAINING EFFECT

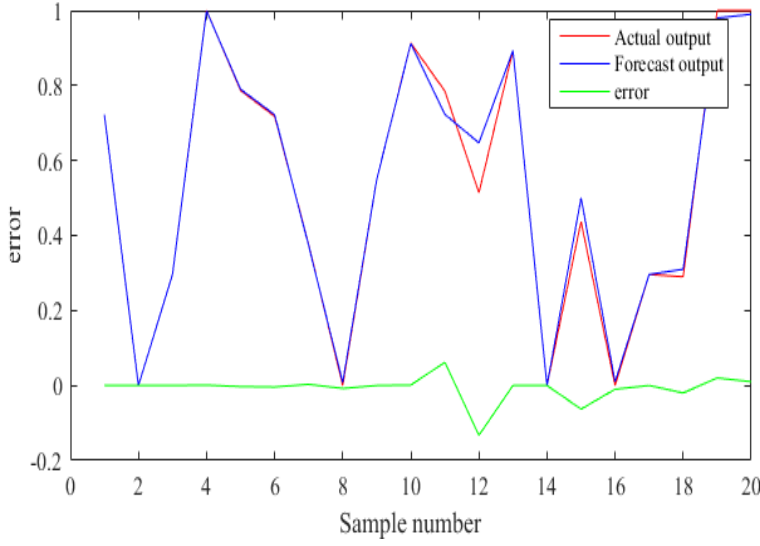

FIGURE VIII. PREDICTION RESULTS

From the above training results, we can see that compared with the prediction results of BP neural network and adaptive fuzzy neural network, the results of adaptive neural network prediction are more accurate. In the process of forecasting, there are some unstable phenomena in the prediction results of $\mathrm{BP}$ neural network, and it is difficult to determine the number of hidden layer and hidden layer nodes. The determination of network structure still needs to be based on experience. The adaptive fuzzy neural network constructed by adaptive fuzzy system and neural network can adaptively determine the most suitable number of hidden layer and hidden layer nodes.

\section{CONCLUSION}

In this paper, the original data of DCPA and TCPA are used to calculate the collision risk of the ship using BP neural network and adaptive fuzzy neural network. Using the original data enables the network training to cover various knowledge of collision risk of the ship. By comparing the results of the BP neural network and the fuzzy neural network, the prediction accuracy of the fuzzy neural network is higher than that of the BP neural network.

\section{ACKNOWLEDGEMENTS}

The National Natural Science Foundation of China (No.51179019, 51090030); Liaoning Provincial Department of Education Key Laboratory Project (No.LZ2015006); Fundamental Research Funds of the Central University for Special Funded Projects (No.3132014022); Liaoning Natural Science Foundation Project (No.20170540098).

\section{REFERENCES}

[1] BI Xiu-ying.Study on Ship's Collision Risk Index and Collision Avoidance Model [D].Dalian Maritime University,2000.

[2] Zhang Ze-hu, Liu De-xin. The research of the ship collision risk model based on fuzzy comprehensive evaluation[D].Dalian Maritime University.2000.

[3] CHEN Xi-de,LIU Zu-yuan. Calculating ship collision risk in open water with improved BP neural network [J]. Ship \& Ocean Engineering,2001(S2):91-93.

[4] ZHU Xiao-lin,XU Han-zhen. The Ship Collision Risk Model Based on Neural Network [J].Journal of Huazhong University of Science and Technology, 2000(10):52-54. 
[5] ZHEGN Zhong-yi, WU Zhao-lin. Fuzzy Decision for Ship Collision Avoidance [J]. Journal of Dalian Maritime University,1996(02):5-8

[6] XU Wen, HU Jiang-qiang,YIN Jian-chuan, et al. Composite evaluation of ship collision risk index based on fuzzy theory[J].Ship Science and Technology,2017,39(13):78-84.

[7] CHEN Jian-hua, CHEN Hong-wei, LIU Ke. A method of estimating ship collision risk based on fuzzy neural network [J].Ship Science and technology.2008 (02):135-138.

[8] CHEN Xu-juan. The Research on Theory and Method of Vessel Collision Avoidance Base on the Fuzzy BP Neural Network [D].HUNAN University,2005.

[9] YANG Guo-bin. Vessels' Collision Risk Index Based on Neural Network with Genetic Algorithm [D]. HeBei University of Technology,2006.

[10] SHI Xin-min,HAO Zheng-qing. Fuzzy Control and MATLAB Simulation [M].Beijing: Tsinghua University Press.2008, 219-254.

[11] LIU Jin-kun. Intelligent Control (Third Edition)[M].Beijing: Publishing House of Electroning Industry . 2014, 126-132.

[12] WANG Xiao-chuan, SHI Feng,YU Lei, et al. MATLAB neural network 43 case studies[M].Beijing: Beijing University Press.2013,288-297. 